\title{
On geometrical alignment properties of two-dimensional forced turbulence
}

\author{
B. Protas ${ }^{\mathrm{a}, \mathrm{b}, *}$, A. Babiano ${ }^{\mathrm{c}}$, N.K.-R. Kevlahan ${ }^{\mathrm{c}, \mathrm{d}}$ \\ ${ }^{a}$ Department of Aerodynamics, Institute of Aeronautics and Applied Mechanics, Warsaw University of Technology, \\ Nowowiejska 24, 00-665 Warsaw, Poland \\ ${ }^{\mathrm{b}}$ Laboratoire de Physique et Mécanique des Milieux Hétérogènes, C.N.R.S., U.R.A. 857, Ecole Supérieure de Physique et Chimie \\ Industrielles, 10, rue Vauquelin, 75231 Paris Cedex 05, France \\ ${ }^{\mathrm{c}}$ CNRS/LMD/ENS, 24 rue Lhomond, 75231 Paris Cedex 5, France \\ ${ }^{\mathrm{d}}$ Department of Mathematics and Statistics, McMaster University, Hamilton, Ontario, Canada L8S 4K1
}

Received 22 July 1998; received in revised form 27 October 1998; accepted 23 November 1998

Communicated by U. Frisch

\begin{abstract}
In the present paper we study some geometrical properties of the small scales in $2 \mathrm{D}$ numerical turbulence. We analyze the alignment of the vorticity gradient with respect to the eigenvectors of the rate of strain tensor, a phenomenon related to the dynamics of the enstrophy cascade. Numerical simulations with different resolutions and dissipation models are used to show non-trivial dependence of the alignment on both the magnitude of the vorticity gradient and the Reynolds number. These findings are shown to be dynamical in origin and imply organization of the small scales in the flow. (C1999 Elsevier Science B.V. All rights reserved.
\end{abstract}

Keywords: 2D turbulence; Vorticity gradient; Geometrical alignments

\section{Introduction}

In the analysis of the behavior of the small scales in turbulence attention is usually focused on the anomalous scaling properties of certain quantities, e.g. velocity increments. It is, however, admitted that this approach is not capable of capturing some of the important characteristics related to the flow structure. At the same time it is believed (cf. [1-3]) that the analysis of geometrical properties of the small scales may yield interesting results concerning the internal organization of the flow. These properties can be analyzed by measuring the alignment of various vector quantities. In the present work we study some geometrical properties of the small scales in two-dimensional (2D) forced turbulence. Such flows are characterized by the presence of large coherent structures which in the course of their non-linear interaction produce and control the strain field. This in turn gives rise to gradual erosion and

${ }^{*}$ Corresponding author. Tel.: +48-22-660-75-23; fax: +48-22-628-25-87; e-mail: bprotas@ meil.pw.edu.pl. 
filamentation of the eddies and thus generates high vorticity gradients which are a generic feature of 2D turbulent dynamics (e.g. [4-6]).

According to [7], stretching and folding of vorticity fronts with different vorticity values is the underlying mechanism of the enstrophy cascade. This process is quantified by the evolution equation for vorticity gradient $\nabla \omega$,

$$
\frac{\mathrm{d}}{\mathrm{d} t} \boldsymbol{\nabla} \boldsymbol{\omega}=\left(\frac{\partial}{\partial t}+\boldsymbol{V} \cdot \boldsymbol{\nabla}\right) \nabla \boldsymbol{\omega}=-(\boldsymbol{\nabla} \boldsymbol{V})^{\mathrm{T}} \cdot \nabla \boldsymbol{\omega}+v \Delta(\boldsymbol{\nabla} \boldsymbol{\omega})
$$

where $(\nabla \boldsymbol{V})^{\mathrm{T}}$ denotes the transpose of the velocity gradient. In the above relation we have accounted for viscosity, nonetheless, in the following analysis its effects will be neglected. Its role is to limit the growth of vorticity gradients. Viscosity therefore becomes important at later stages of the evolution. We are, however, interested in the non-linear amplification of $\nabla \omega$, which is essentially an inviscid phenomenon.

Vorticity gradient points in the direction of the steepest increase of vorticity. An equivalent description can be cast in terms of palinstrophy $\boldsymbol{\eta}=\boldsymbol{\nabla} \times(\omega \boldsymbol{k})$ which is the dual vector with respect to vorticity gradient and is tangent to the isolines of vorticity. Its evolution is governed by the following equation

$$
\frac{\mathrm{d}}{\mathrm{d} t} \boldsymbol{\eta}=\left(\frac{\partial}{\partial t}+\boldsymbol{V} \cdot \boldsymbol{\nabla}\right) \boldsymbol{\eta}=\boldsymbol{\eta} \cdot \boldsymbol{\nabla} \boldsymbol{V}+v \Delta \boldsymbol{\eta}
$$

We will recall below that the evolution of the vorticity gradient, and consequently of the enstrophy cascade, depends on the properties of the $\nabla V$ tensor and on the alignment of $\nabla \omega$ with respect to the eigenvectors of the symmetric part of $\boldsymbol{\nabla} \boldsymbol{V}$. Multiplying Eq. (1) by $(\nabla \boldsymbol{\omega})^{\mathrm{T}}$, integrating the resulting relation over the flow domain, and then assuming statistical stationarity and homogeneity, we obtain

$$
-\left\langle(\nabla \omega)^{\mathrm{T}}(\nabla \boldsymbol{V})^{\mathrm{T}} \nabla \boldsymbol{\omega}\right\rangle=v\left\langle(\nabla \nabla \omega)^{2}\right\rangle,
$$

where \langle\rangle denotes spatial average and the viscous term was transformed using integration by parts. Since the right-hand side term is positive definite, this relation implies that the net production of vorticity gradient by the non-linear effects is always positive. Evidence will be presented below for some non-trivial characteristics of this phenomenon in 2D forced turbulence. We will use data from the direct numerical simulation (DNS) of the Navier-Stokes equations with periodic boundary conditions and with both Newtonian and hyperviscous dissipation models. The reason for this is that hyperviscosity is used to model a high Reynolds number flow, whereas the Newtonian viscosity enables one to check whether the former introduces spurious effects. Qualitatively, the Newtonian and hyperviscous dissipation models may be regarded as representing the low and high Reynolds numbers, respectively. At the same time, we will consider the results of simulations with different resolutions corresponding to different Reynolds numbers. Forcing will be chosen so as to assure that the enstrophy cascades in the different simulations are consistent with one another, with their extent depending on the Reynolds number.

Vorticity gradient is an active vector and its evolution involves an interplay between kinematics and dynamics. In [8] it was shown that certain alignments may be largely kinematic in origin. This means that even random Gaussian fields may have some non-trivial geometric properties. In order to distinguish between the kinematic and dynamic alignments we will compare our fields with their random counterpart. The latter is obtained by randomizing the phases of the coefficients in the Fourier transformed vorticity field, while retaining the amplitudes of the coefficients, so that the energy spectrum is not affected.

The organization of the paper is as follows. In the next Section we derive a set of diagnostics that we later use to characterize the alignments. In Section 3 we give some details concerning the numerical simulations and present the results. In Section 4 we will discuss the results and make some final remarks. 


\section{Characterization of geometrical alignments}

Eq. (1) combines the Lagrangian derivative with Eulerian gradients, therefore, following a fluid particle, the vorticity gradient evolves according to the local properties of the term $-(\nabla \boldsymbol{V})^{\mathrm{T}} \cdot \nabla \boldsymbol{\omega}$. Transfer of vorticity towards smaller scales of motion known as the enstrophy cascade is the result of the non-linearity of this term. We will now assess the efficiency of $-(\boldsymbol{\nabla} \boldsymbol{V})^{\mathrm{T}} \cdot \nabla \boldsymbol{\omega}$ as regards production of vorticity gradient. In [9,10] and then in [11] it was argued that $\nabla V$ varies slowly comparing to the changes of $\nabla \omega$ and may thus be considered frozen. This is equivalent to the assumption of scale separation and allows one to linearize Eq. (1) around a fixed value of $\boldsymbol{\nabla} \boldsymbol{V}$. On the other hand, in [12] it was shown that this linearization is only valid in a small fraction of the flow domain. Then in [13] the authors considered the second order in time equation for the particle dispersion and showed how the time variation of the velocity gradient can be taken into account to modify the above analysis. However, in our investigation we are concerned with space averages of instantaneous intensities of vorticity gradient production. In this sense, we do not have to resort to linearization in order to compute the magnitude of the non-linear term. Consequently, the question of how long this linearization remains valid is not relevant here. Further discussion of issues related to persistence of strain can be found in [14,15] and references quoted therein.

The transpose of the velocity gradient $(\boldsymbol{\nabla} \boldsymbol{V})^{\mathrm{T}}$ can be rewritten in terms of strain and vorticity,

$$
(\nabla \boldsymbol{V})^{\mathrm{T}}=\frac{1}{2}\left[\begin{array}{cc}
s_{11} & s_{12}+\omega \\
s_{12}-\omega & -s_{11}
\end{array}\right],
$$

where $s_{11}=2(\partial u / \partial x)=-2(\partial v / \partial y), s_{12}=(\partial u / \partial y)+(\partial v / \partial x)$ and $\omega=(\partial v / \partial x)-(\partial u / \partial y)$. Its eigenvalues are given by

$$
\lambda_{ \pm}= \pm \frac{1}{2} \sqrt{s_{11}^{2}+s_{12}^{2}-\omega^{2}}
$$

In [10] it was shown that according to whether the eigenvalues $\lambda$ are purely real or purely imaginary, one can distinguish two dynamically distinct behaviors of vorticity gradient. If $\lambda$ is real, then exponential growth of vorticity gradient may take place. It will be shown below that the actual growth rate depends on the alignment of $\nabla \omega$ with respect to the eigenvectors of the symmetric part of $\boldsymbol{\nabla} \boldsymbol{V}$. Clearly, these regions contribute to the enstrophy cascade. On the other hand, if the eigenvalues $\lambda$ are imaginary, the vorticity gradient rotates with periodic amplification and damping, a mechanism which leads to the the elliptic instability [16]. According to the relative importance of strain and vorticity, the above regimes are labeled hyperbolic and elliptic, respectively. Elliptic regions are passive as regards the enstrophy cascade and thus the following analysis focuses exclusively on the hyperbolic parts of the flow domain.

We now proceed to quantify the alignment characteristics. Stretching is due exclusively to the strain tensor $\boldsymbol{D}=(1 / 2)\left(\nabla \boldsymbol{V}+\nabla \boldsymbol{V}^{\mathrm{T}}\right)$. Its eigenvectors are orthogonal and represent the directions of maximum stretching and compression,

$$
\boldsymbol{d}_{1}=\left[\begin{array}{c}
s_{12} \\
\sqrt{s_{11}^{2}+s_{12}^{2}}-s_{11}
\end{array}\right], \quad \boldsymbol{d}_{2}=\left[\begin{array}{c}
-s_{12} \\
\sqrt{s_{11}^{2}+s_{12}^{2}}+s_{11}
\end{array}\right] .
$$

The following two parameters can be introduced to characterize the alignment properties of vorticity gradient [17]:

- instantaneous production rate of vorticity gradient $\kappa$

$$
\kappa=\frac{\mathrm{d} \ln |\nabla \omega|}{\mathrm{d} t}=-\boldsymbol{m}^{\mathrm{T}} \boldsymbol{D} \boldsymbol{m},
$$

where $\boldsymbol{m}=\nabla \omega /|\nabla \omega|$, 
Table 1

Summary of the numerical parameters used in the simulations

\begin{tabular}{llll}
\hline & H128 & H512 & H1728 \\
\hline (hyper) viscosity & $5 \times 10^{-5}$ & $1.75 \times 10^{-5}$ & $5 \times 10^{-6}$ \\
time step $\delta t$ of integration & $10^{-4}$ & $2.5 \times 10^{-5}$ & $8 \times 10^{-3}$ \\
forcing wavenumber & 10 & 40 & $10^{-5}$ \\
spectral slope in the enstrophy range & -4.0 & -4.0 & 40 \\
\hline
\end{tabular}

Table 2

Spatially averaged characteristics of the fields under consideration. See text for definitions of $\left\langle h^{+}\right\rangle,\langle\kappa\rangle$ and $\langle e\rangle$. The statistics are computed in the hyperbolic parts of the flow domain which were extracted using Eq. (5).

\begin{tabular}{lcccc}
\hline & H128 & H512 & H1728 & N1728 \\
\hline$\left\langle h^{+}\right\rangle$ & 0.613 & 0.632 & 0.673 & 0.558 \\
$\langle\kappa\rangle$ & 10.33 & 84.91 & 88.23 & 31.26 \\
$\langle e\rangle$ & 0.12 & 0.14 & 0.18 & 0.501 \\
\hline
\end{tabular}

- normalized instantaneous production rate of vorticity gradient $e$

$$
e=\frac{-\boldsymbol{m}^{\mathrm{T}} \boldsymbol{D} \boldsymbol{m}}{(\boldsymbol{D}: \boldsymbol{D})^{1 / 2}}
$$

It can be easily verified that in our case

$$
\kappa=\frac{1}{2} \sqrt{s_{11}^{2}+s_{12}^{2}} \cos (2 \alpha)
$$

where $\alpha$ is the angle between the vorticity gradient $\nabla \omega$ and the compressing eigenvector $d_{2}$. Similarly,

$$
e=\frac{\sqrt{2}}{2} \cos (2 \alpha) .
$$

One can see that $\kappa$ represents combined effects of strain magnitude and geometrical alignment, whereas $e$ relates to alignment only.

It should be emphasized that the role played by the rotation tensor $\boldsymbol{\Omega}=(1 / 2)\left(\nabla \boldsymbol{V}-\boldsymbol{\nabla} \boldsymbol{V}^{\mathrm{T}}\right)$ is different in stretching of vorticity (3D) and vorticity gradients (2D). Normally one has $\mathbf{n}^{\mathrm{T}} \boldsymbol{\Omega} \boldsymbol{n} \equiv 0$ for any vector $\boldsymbol{n}$, which implies that the rotation tensor $\Omega$ does not contribute to stretching and is responsible exclusively for reorientation of the stretched vector. In the case of stretching of vorticity, one always has $\Omega \omega \equiv 0$ which means that vorticity vector is unaffected by the antisymmetric part of the velocity gradient tensor.

It was argued in [8] that in the context of 3D flows the angle $\gamma_{\omega}=\angle(\boldsymbol{D} \boldsymbol{\omega}, \boldsymbol{\omega})$ between vorticity and the so called 'vortex stretching' vector has particularly interesting properties. In our case the equivalent would be $\gamma_{\nabla \omega}=$ $\angle(\boldsymbol{D} \nabla \boldsymbol{\omega}, \nabla \boldsymbol{\omega})$. It is however straightforward to show that $\gamma_{\nabla \omega}=2 \alpha$ and therefore there is no point in analyzing it separately. From the relation (10) it is clear that build-up of vorticity gradient takes place when it lies within the range of $\pi / 4$ from the principal direction of compression $\boldsymbol{d}_{\mathbf{2}}$. In [18] 2D skewness $S_{2}$ was introduced to assess the net production of vorticity gradient. It is directly related to our parameter $\langle e\rangle$, the only difference being that the definition of $S_{2}$ relies on the assumption that the components of the vorticity gradient and the strain tensor are locally isotropic. This simplification does not appear justified in the context of our study, since we are interested in the small scale details of the mechanism leading to amplification of vorticity gradient, in particular in its spatial structure. Finally, it should be noted that $\boldsymbol{D}, \kappa$ and $e$ are all Galilean invariants. 


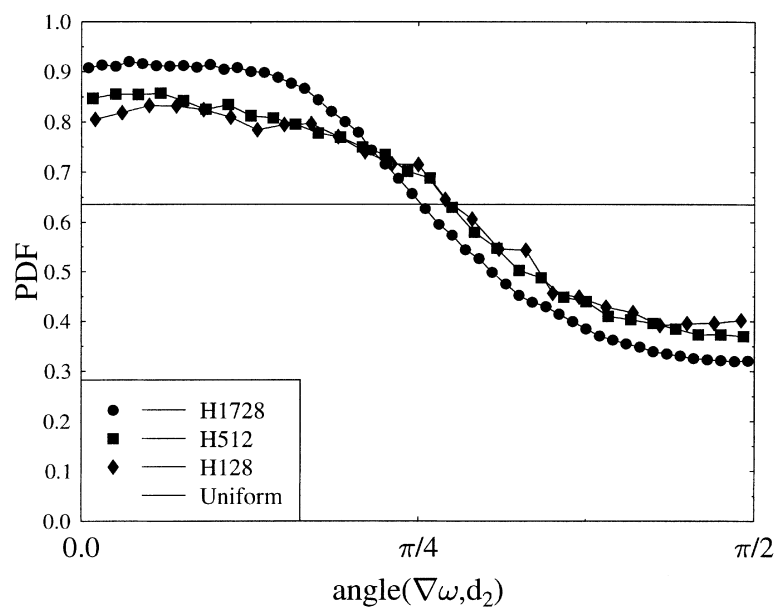

Fig. 1. PDFs of the alignment angle $\alpha$ between the vorticity gradient $\nabla \omega$ and the compressing eigenvector $\boldsymbol{d}_{2}$ for the hyperviscous fields with different resolutions.

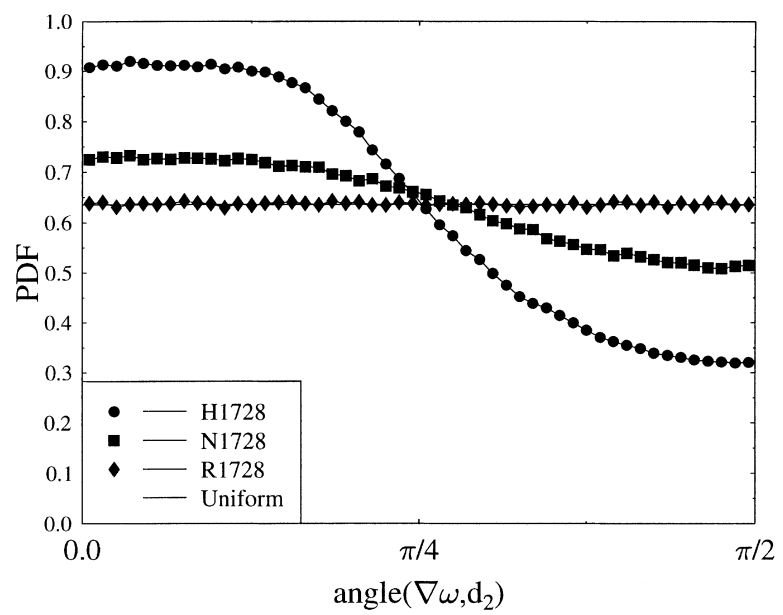

Fig. 2. PDFs of the alignment angle $\alpha$ between the vorticity gradient $\nabla \omega$ and the compressing eigenvector $\boldsymbol{d}_{2}$ for the random field and the fields with different dissipation models.

\section{Results of the numerical simulations}

We apply the diagnostics introduced above to fields obtained from the numerical simulation of 2D forced turbulence in a periodic domain. The Navier-Stokes equations are solved using the pseudo-spectral method developed in [19]. For the reasons mentioned in the introduction we performed simulations with both Newtonian and hyperviscous dissipation models. The dissipative term used in the latter case was $v \Delta^{8} \omega$ (i.e. $-v k^{16} \hat{\omega}$ ). In order to assure stationarity, dissipation also includes a term representing large-scale linear friction. The hyperviscous simulations were carried out with the resolutions $128^{2}, 512^{2}$ and $1728^{2}$. These sets of data will be referred to as H128, H512 and H1728, respectively. The last field was also used to obtain the randomized counterpart which we will denote R1728. The system with the Newtonian dissipation was simulated with the resolution $1728^{2}$ and will be labeled N1728. In the runs H512, H1728 and N1728 forcing was applied at the wavenumber $k=40$, whereas in the case H128, 


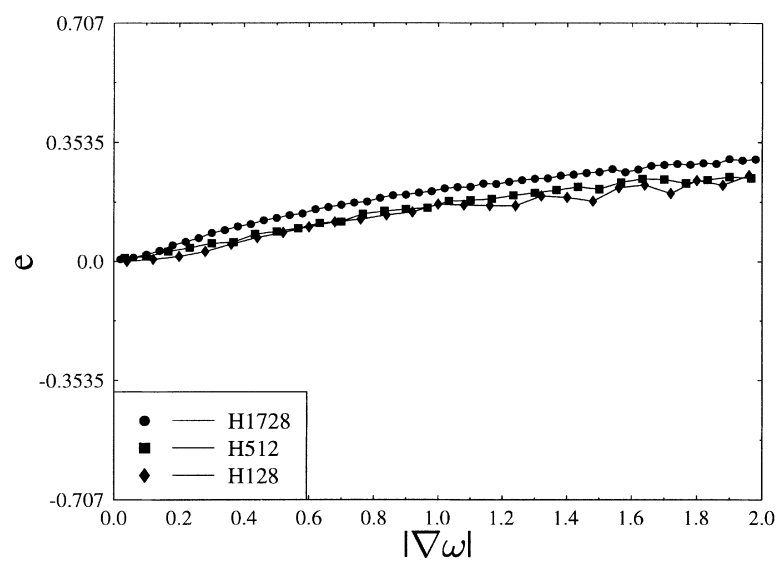

Fig. 3. Normalized production rate of vorticity gradient $e$ versus magnitude of $|\nabla \omega|$ for the hyperviscous fields with different resolutions. Here, $|\nabla \omega|$ is normalized with respect to its mean value in the hyperbolic parts of the flow.

the corresponding wavenumber was $k=10$. In the latter case forcing was shifted towards smaller wavenumbers in order to ensure consistency of the enstrophy cascades obtained in the simulations with different resolutions. In all cases forcing was effected by keeping the amplitude of the given wavevector at a prescribed constant value. In Table 1 we summarize all the numerical parameters of the simulations. In computational studies of 2D turbulence with periodic boundary conditions, no good definition of the Reynolds number is available, particularly when the hyperviscous dissipation is used. The Reynolds number may be related to the extent of the inertial ranges which can be qualitatively estimated as the ratio $k_{\max } / k_{\min }$, where $k_{\max }$ represents the dissipation wavenumber and $k_{\min } \approx 1$. In this respect, hyperviscosity will always result in a higher Reynolds number, as in this case dissipation is limited to smaller scales. All the analyzed fields correspond to times when a state of the statistical equilibrium was reached.

We begin the presentation of the results with a summary of the field averaged characteristics which are related to the net production of vorticity gradient. The fields H512, H1728, N1728 and R1728 consist of enough points to produce converged statistics. In the case of N128 the averages were computed using the data from ten different fields corresponding to ten instances of time sufficiently separated from one another so as to prevent any correlation between them. All the averages, $\left\langle h^{+}\right\rangle,\langle\kappa\rangle$ and $\langle e\rangle$, were restricted to the hyperbolic parts of the flow domain which were identified using the instantaneous values of the eigenvalue $\lambda$ (cf. Eq. (10)). In Table 2 one can note that the production of vorticity gradients is always positive (cf. Eq. (3)) and that they are produced more effectively with increasing Reynolds numbers (corresponding to increasing resolution). This concerns the values of the normalized efficiency $\langle e\rangle$ (cf. Eq. (10)), as well as the fraction $\left\langle h^{+}\right\rangle$of the hyperbolic regions where build-up of $\nabla \omega$ occurs. The latter implies that the area of regions with amplification of vorticity gradients is bigger than that corresponding to damping and increases with the Reynolds number. The Newtonian field (N1728) which represents an effectively much smaller Reynolds number is characterized by less efficient production of vorticity gradient than its hyperviscous counterpart (H1728). The random field (R1728) is neutral in this respect. There is some discrepancy as regards the values of the parameter $\langle\kappa\rangle$, i.e. the values corresponding to the cases H128 and N1728 are not consistent with those obtained for $\mathrm{H} 512$ and H1728. This results from the fact that $\langle\kappa\rangle$ is not a non-dimensionalized quantity and that as such it depends on the large scale characteristics of the flow. These are in turn different for different flows, depending on the particular kind of forcing and large-scale dissipation. Consequently, $\langle\kappa\rangle$ does not appear to be an objective criterion for quantifying alignments and in all further considerations the normalized instantaneous production rate $\langle e\rangle$ will be used. 


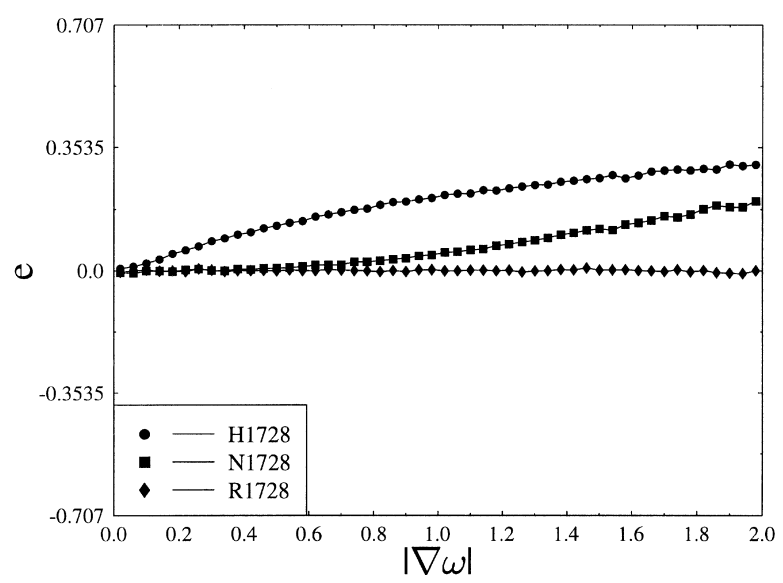

Fig. 4. Normalized production rate of vorticity gradient $e$ versus magnitude of $|\nabla \omega|$ for the random field and the fields with different dissipation models. Here, $|\nabla \omega|$ is normalized with respect to its mean value in the hyperbolic parts of the flow.

Now we proceed to analyze the statistics of the geometrical alignments. In Figs. 1 and 2 we show the PDFs of the angle $\alpha$ (cf. Eq. (10)) for the hyperviscous fields with different resolutions and for the fields with the highest resolution and different dissipation models. All of them (apart from the random field) reveal a tendency for preferential alignment which increases with the Reynolds number (equivalently the resolution). The field N1728 corresponding to the smallest Reynolds number is characterized by the weakest alignments. The random field R1728 does not reveal any alignment whatsoever. At this point it is necessary to remark that the PDF of the cosine of an angle $\alpha$ instead of the PDF of the angle itself could be misleading. For a uniform distribution of angles, the corresponding PDF of the cosine is not uniform; moreover, it is unbounded at the limits of the range (like $1 / \sin (\alpha) \simeq 1 / \alpha$ for $\alpha \rightarrow 0$ ).

Next we investigate how the alignment properties of the vorticity gradient change with its magnitude. In Figs. 3 and 4 we show the average values of the normalized efficiency $e$ corresponding to different values of $|\nabla \omega|$, again for fields with different resolutions and different dissipation models (here $|\nabla \omega|$ is normalized with respect to its mean value in the hyperbolic parts of the flow). One can see that in all cases more efficient alignments are correlated with substantial magnitudes of $|\nabla \omega|$. In other words, the tendency for the alignment with the compressing eigenvector $\boldsymbol{d}_{2}$ increases with the steepness of the vorticity gradient. Similar to what was observed in the case of the PDFs, this effect becomes less pronounced as the Reynolds number is decreased and is weakest for the field with the Newtonian dissipation model. Interestingly, one can observe (Fig. 4) that there is a qualitative difference between the cases H1728 and N1728 as regards the relation $e$ versus $|\nabla \omega|$ : the two curves are characterized by different convexities. Furthermore, in the field N1728 the average production of the vorticity gradient is close to zero for small magnitudes of $|\nabla \omega|$. In Figs. 5 and 6 we present the PDFs of the alignment angle $\alpha$ conditioned to different values of $|\nabla \omega|$ for the fields H1728 and N1728, respectively. One can again note the bias for preferential alignment which becomes more evident as the magnitude of $|\nabla \boldsymbol{\omega}|$ increases. Finally, in Figs. 7 and 8 we show the stretching eigenvectors $\boldsymbol{d}_{1}$ overlaid on the corresponding vorticity fields for the hyperviscous (H1728) and Newtonian (N1728) cases, respectively. The eigenvectors are drawn only in the hyperbolic parts of the flow domain. The reason for showing $\boldsymbol{d}_{1}$, rather than $\boldsymbol{d}_{2}$, will be made clear below. Note that the Newtonian field is more blurred compared to the hyperviscous one which corresponds to an effectively lower Reynolds number. 


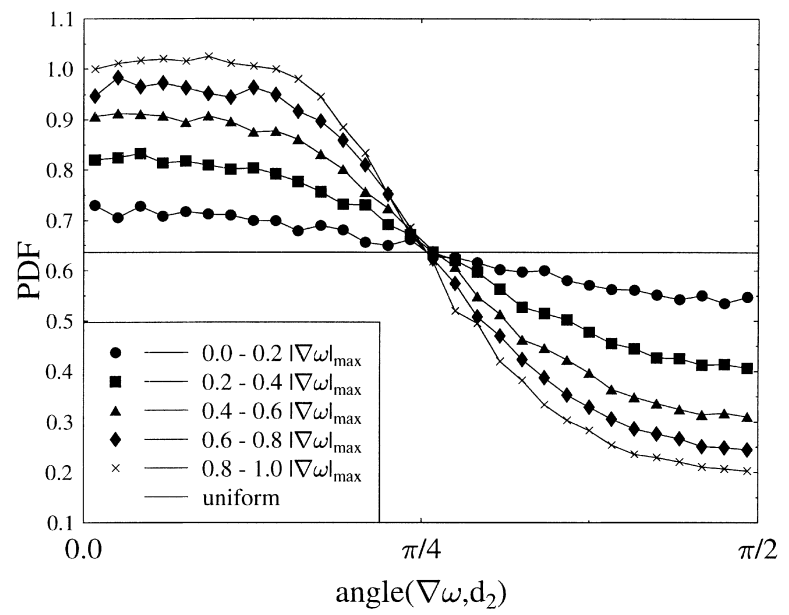

Fig. 5. PDFs of the alignment angle $\alpha$ (refer Eq. (10)) conditioned to different values of $|\nabla \omega|$ for the field H1728.

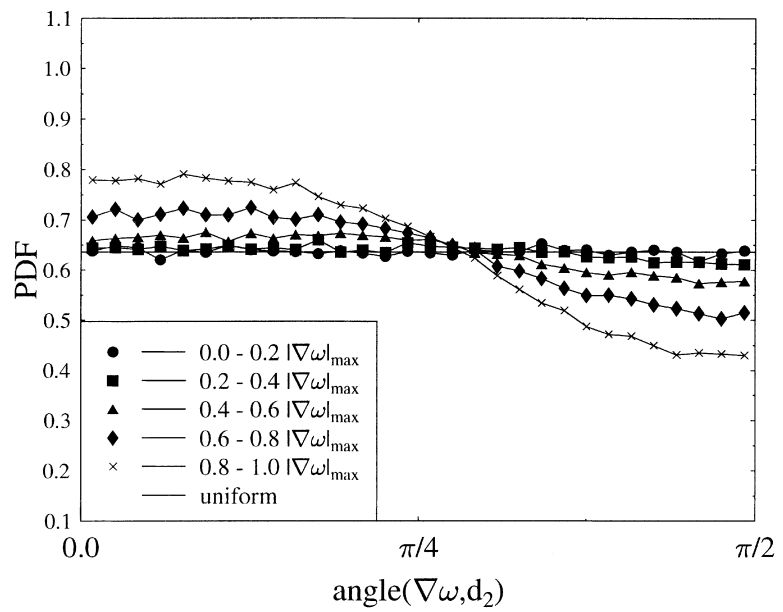

Fig. 6. PDFs of the alignment angle $\alpha$ (refer Eq. (10)) conditioned to different values of $|\nabla \omega|$ for the field N1728.

\section{Conclusions and final remarks}

We shall now summarize our main results. The first observation is that in all the fields the vorticity gradient reveals a tendency for the alignment with the compressing eigenvector $\boldsymbol{d}_{2}$ of the strain tensor which results in its amplification. This is however a direct consequence of relation (3). The most important finding is that this alignment becomes less pronounced as the Reynolds number is decreased and is weakest in the field with the Newtonian dissipation model. Moreover, it was observed that the alignment increases with the magnitude of $|\nabla \omega|$ with some qualitative differences between the hyperviscous and Newtonian dissipation models (in the Newtonian field there is no preferential alignment for $|\nabla \omega|<0.6$ at all). These characteristics also appear to depend on the Reynolds number.

The results may be explained physically using the dual representation in terms of palinstrophy $\boldsymbol{\eta}$ (cf. Eq. (2)). The vector $\boldsymbol{\eta}$ is always directed along the axis of the filament and amplification of $\boldsymbol{\eta}$ thus corresponds to elongation of the filament. Evolution of $\boldsymbol{\eta}$ is described by the left eigenvectors of $\boldsymbol{D}$ and the alignment angle $\alpha$ may equivalently be interpreted as the angle between the palinstrophy vector $\boldsymbol{\eta}$ and the stretching eigenvector $\boldsymbol{d}_{1}$. Our results therefore 


\section{Vorticity Field with Stretching Eigenvectors}

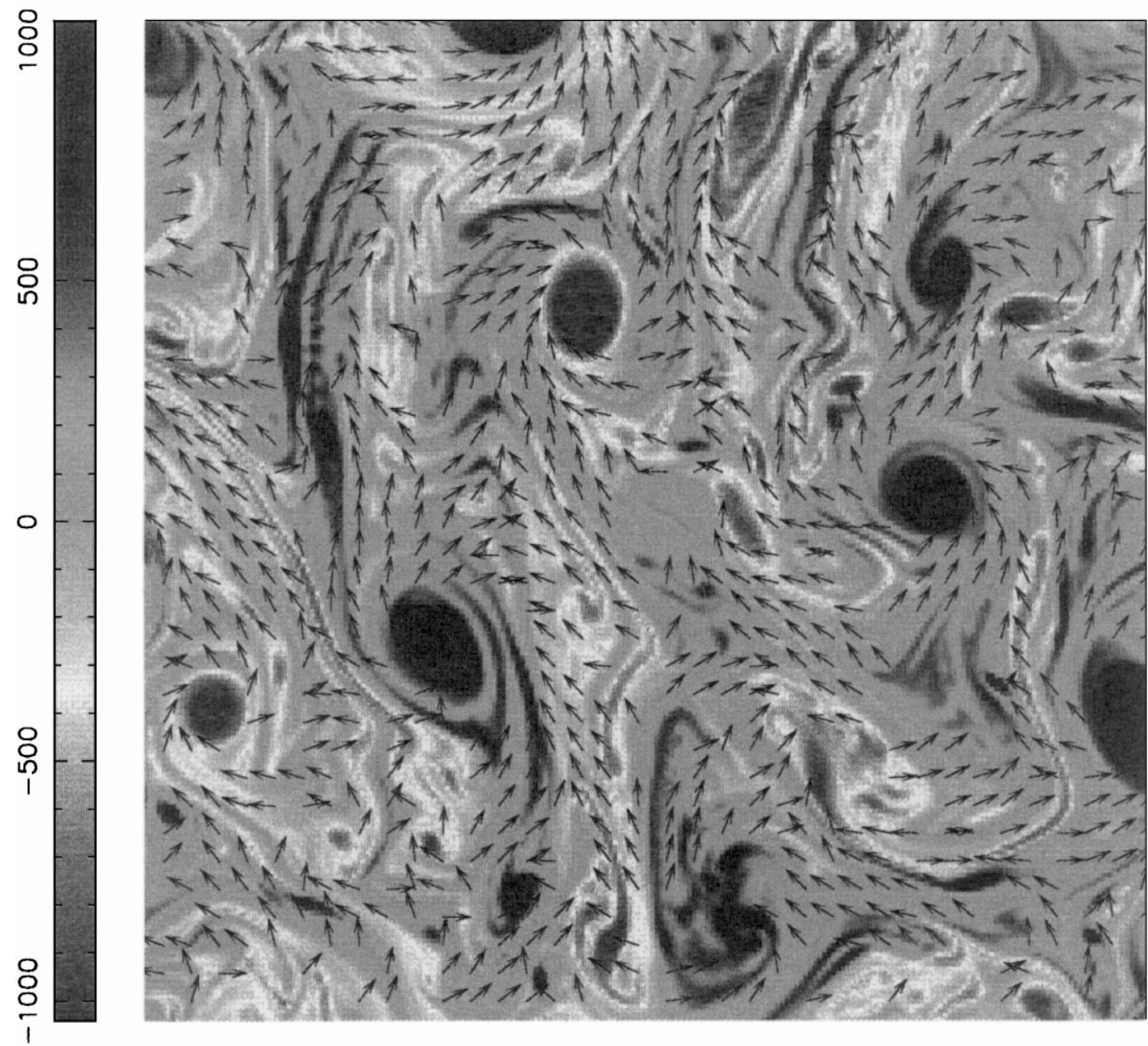

Fig. 7. The stretching eigenvectors $\boldsymbol{d}_{1}$ overlaid on the corresponding vorticity field for the hyperviscous (H1728) dissipation model. The eigenvectors are shown only in the hyperbolic parts of the flow domain. The figure presents a $216^{2}$ segment of the whole field.

imply that with increasing Reynolds number the vorticity filaments are more aligned with the local direction of stretching. The same happens when the filaments become thinner and more elongated. These effects can be observed in Figs. 7 and 8, notably for the case of filaments which are detached from the large vortices and form the background flow. Conversely, some filaments remain bound to the eddies from which they were stripped and reveal no preferential alignment. This is because, for a single concentrated vortex, the rate of strain eigenvectors form the angle $\pm \pi / 4$ with respect to the circular filaments that surround the vortex. This explains the neutral alignments which are apparent for some of the bound filaments (Figs. 7 and 8). The random field R1728 does not reveal any alignments which might be the case if they were kinematic in origin. This indicates that the alignments that were found are a dynamic property of the turbulent flows.

In [7], it was argued that the enstrophy cascade essentially consists in stretching and folding of vorticity layers. The effectiveness of this process crucially depends on how the filaments are aligned with respect to the external 


\section{Vorticity Field with Stretching Eigenvectors}

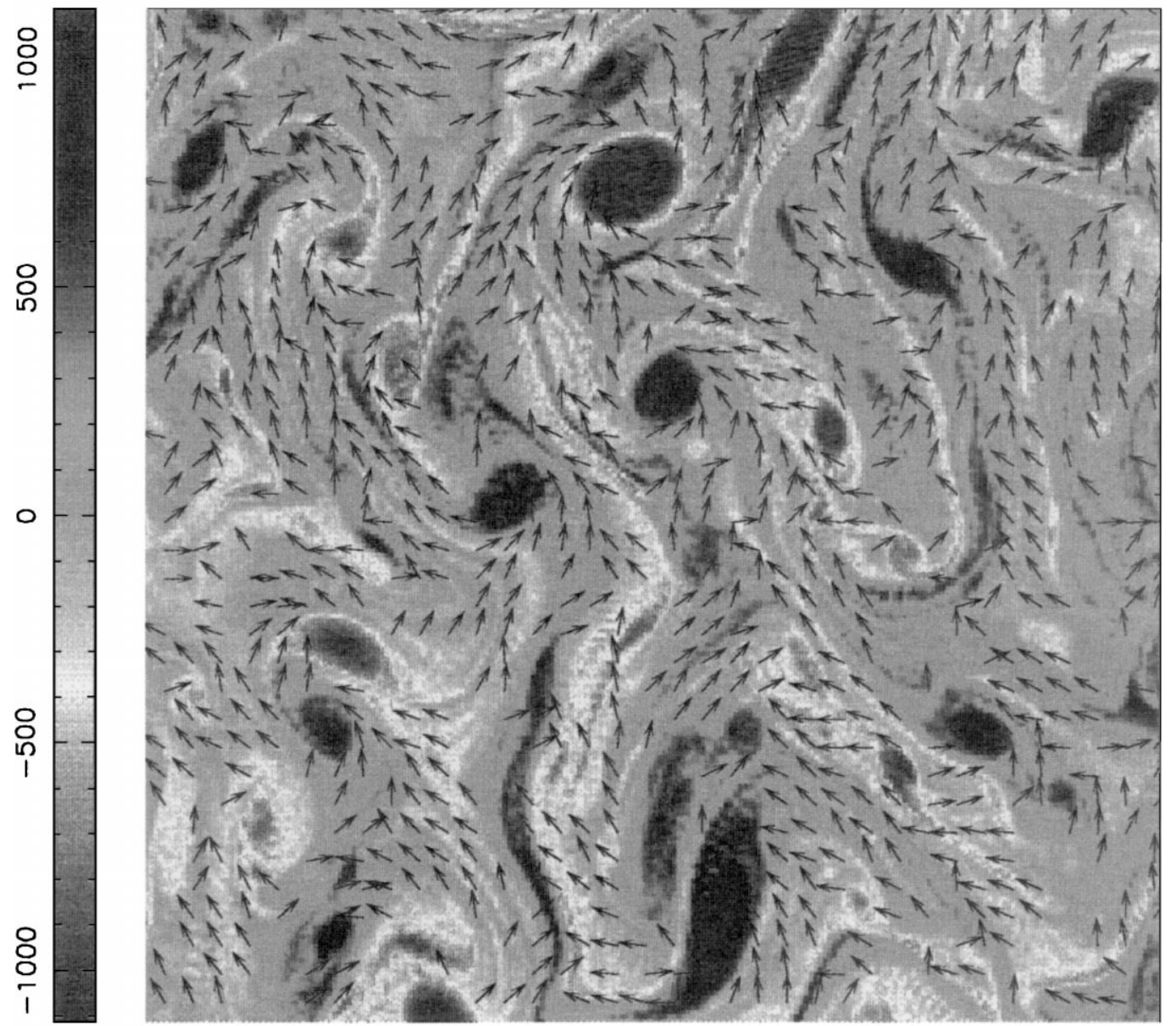

Fig. 8. The stretching eigenvectors $\boldsymbol{d}_{1}$ overlaid on the corresponding vorticity field for the Newtonian (N1728) dissipation model. The eigenvectors are shown only in the hyperbolic parts of the flow domain. The figure presents a $216^{2}$ segment of the whole field.

straining field. We showed evidence that these characteristics vary systematically with the Reynolds number and may also depend on the dissipation model. At this point it should be emphasized that the geometrical properties of the small scales may only be studied in the physical space. Such effects cannot be captured in the Fourier space, since then all the geometrical structure is lost.

The main conclusion of this investigation is that vorticity filaments become more passive as the Reynolds number increases. In Figs. 2 and 4 some differences between the hyperviscous and the Newtonian fields are evident. However, it remains an open question whether they are due to the form or due to the magnitude of the dissipation term.

\section{Acknowledgements}

B.P. was supported by the Foundation for Polish Science. Computations were performed at IDRIS under the Contract No. 940338. We are grateful to A. Tsinober, A. Styczek and J. Szumbarski for their valuable comments. 


\section{References}

[1] A. Tsinober, Eur. J. Mech. B/Fluids 17(4) (1998) 421.

[2] P. Constantin, SIAM Review 36 (1994) 73.

[3] W.T. Ashurst, A.R. Kerstein, R.M. Kerr, C.H. Gibson, Phys. Fluids 30(8) (1987) 2343.

[4] M.V. Melander, J.C. McWilliams, N.J. Zabusky, J. Fluid Mech. 178 (1987) 137.

[5] H.B. Yao, N.J. Zabusky, D.G. Dritschel, Phys. Fluids 7 (1995) 539.

[6] B. Legras, D. Dritschel, Appl. Sci. Res. 51 (1993) 445.

[7] P.G. Saffman, Stud. Appl. Math. 50 (1971) 377.

[8] L. Shtilman, M. Spector, A. Tsinober, J. Fluid Mech. 247 (1993) 65.

[9] J. Weiss, Report LJI-TN-81-121, La Jolla Inst., San Diego, CA, 1981.

[10] J. Weiss, Physica D 48 (1991) 273.

[11] M.E. Brachet, M. Meneguzzi, H. Politano, P.L. Sulem, J. Fluid Mech. 194 (1988) 333.

[12] C. Basdevant, T. Philipovitch, Physica D 73 (1994) 17.

[13] B.L. Hua, P. Klein, Physica D 113 (1998) 98.

[14] M. Tabor, I. Klapper, Chaos, Solitons and Fractals 4(6) (1994) 1031.

[15] E. Dresselhaus, M. Tabor, J. Fluid Mech. 236 (1991) 415.

[16] B.J. Bayly, Phys. Rev. Lett. 57(17) (1986) 2160.

[17] J.M. Ottino, The Kinematics of Mixing: Stretching, Chaos and Transport, Cambridge University Press, Cambridge, 1995.

[18] J.R. Herring, S.A. Orszag, R.H. Kraichnan, D.G. Fox, J. Fluid Mech. 66 (1974) 417.

[19] C. Basdevant, B. Legras, R. Sadourny, M. Beland, J. Atm. Sci. 38 (1981) 2305. 